\title{
Insolubility from No-Signalling
}

\author{
Guido Bacciagaluppi*
}

9 January 2013

\begin{abstract}
This paper improves on the result in my Bacciagaluppi (2013), showing that within the framework of the unitary Schrödinger equation it is impossible to reproduce the phenomenological description of quantum mechanical measurements (in particular the collapse of the state of the measured system) by assuming a suitable mixed initial state of the apparatus. The result follows directly from the nosignalling theorem applied to the entangled state of measured system and ancilla. As opposed to other 'insolubility theorems' for the measurement problem of quantum mechanics, it focuses on the impossibility of reproducing the phenomenological collapse of the state of the measured system.
\end{abstract}

Phenomenologically, a quantum measurement consists of a transformation of the state of a quantum system (the 'collapse' of the state) upon interaction with a suitable measuring apparatus. In each experimental setup ('measurement'), one of a family of transformations can take place, with a characteristic probability distribution determined by the quantum state of the system (the 'Born rule').

*Department of Philosophy, University of Aberdeen, and Institut d'Histoire et de Philosophie des Sciences et des Techniques (CNRS, Paris 1, ENS). Address for correspondence: Department of Philosophy, University of Aberdeen, Old Brewery, High Street, Aberdeen AB24 3UB, Scotland, U.K. (e-mail: g.bacciagaluppi@abdn.ac.uk). 
The theoretical description of such experiments relies on an appropriate coupling of the quantum system to an ancillary system, followed by a special application of the collapse and Born rule to the ancilla. In this way, the most general (physically sensible) families of transformations on the state of the quantum system can be implemented. Mathematically, this relies on the Naimark dilation theorem. (See for instance Busch, Grabowsky and Lahti (1995).)

This theoretical description was developed (for special cases) already by von Neumann (1932), and it contains a notorious regress: the application of the phenomenological rules is shifted from the quantum system to be measured to the ancilla; from the ancilla (if the special application of the rules is again modelled theoretically) it is shifted to a 'second-order' ancilla; from thence to a 'third-order' one, and so forth.

It was von Neumann himself who asked (at least for the sake of argument) whether this regress could be stopped if, at some stage, the apparent probabilistic aspect of the evolution could be explained as a statistical effect of coupling our system(s) of interest to some system of which only a coarse-grained description is available (a macrostate). Knowing the exact microstate of this system (and the exact form of the unitary evolution of the composite) would allow us to predict exactly the final state of the composite (say, a product consisting of the 'collapsed' state of the system and any microscopic ancilla(s), and a microstate of the apparatus belonging to a macrostate associated with a definite outcome). Thus, the theoretical description of the measurement process would be continuous and deterministic, but due to our ignorance of the exact microstate of the 'apparatus', the phenomenological one would be discontinuous and indeterministic, as given by collapse and Born rule.

Von Neumann's argument to the effect that this redescription of the measurement process is impossible, and its various later generalisations, have become known as 'insolubility proofs' for the measurement problem of quantum mechanics. The intuitive core of von Neumann's argument is that if the statistical element of the measurement comes from the description of the apparatus, then it will not depend on the state of the system in the way required by the Born rule. 
The later versions of von Neumann's theorem all focus on showing the impossibility in general of obtaining definite measurement results by assuming an initially mixed state for the apparatus. The simplest and most general of these is the one given by Bassi and Ghirardi (2000). (See Brown (1986) and Bacciagaluppi (2013) for further references and for discussion.)

In this paper, I shall provide an equally simple proof of insolubility, but following a different strategy, namely pointing out that no unitary interaction with an ancilla can deterministically steer the measured system to different 'collapsed' states, on pains of violating the quantum mechanical no-signalling theorem (Ghirardi, Rimini and Weber 1980). The result, which improves on my Bacciagaluppi (2013), is an obvious consequence of the no-signalling theorem, but has never to my knowledge been brought out in this form.

In section 1 of the paper, I shall briefly rehearse in somewhat more detail the standard description of measurement based on the dilation theorem, using Stern-Gerlach measurements as an example. In section 2, I shall describe von Neumann's original insolubility argument, and in section 3 spell out my new argument. Brief remarks on related questions conclude the paper (section 4).

\section{Stern-Gerlach measurements}

Let us first take the case of an ideal measurement of spin. A system with both spin and position degrees of freedom is described using the tensor product of the Hilbert spaces used to describe a 'pure' spin- $1 / 2$ system and a spinless particle, just as if one were composing two separate systems.

Take an electron that we assume to be initially in a state

$$
|\psi\rangle \otimes \varphi .
$$

Now suppose we want to perform a measurement of spin in some given direction, and that with respect to this spin basis, $|\psi\rangle=\alpha|+\rangle+\beta|-\rangle$, so that (1) equals

$$
\alpha|+\rangle \otimes \varphi+\beta|-\rangle \otimes \varphi
$$

If we pass the electron through an ideal Stern-Gerlach magnet, we obtain

$$
\alpha|+\rangle \otimes \varphi_{+}+\beta|-\rangle \otimes \varphi_{-}
$$


(where $\varphi_{+}$and $\varphi_{-}$are suitably deflected versions of $\varphi$ ). We see that the spin degree of freedom of the electron is entangled with its position degrees of freedom.

We now detect the electron on a screen, i.e. perform a position measurement on the spatial degrees of freedom of the electron. It is a discretised measurement of position, because we only need to distinguish whether the electron hits the half of the screen associated with the up or down component of the spin. ${ }^{1}$ Assuming that $\varphi_{+}$and $\varphi_{-}$do not overlap, the standard collapse postulate and Born rule, applied to the detection of the electron on the screen, will yield

$$
| \pm\rangle \otimes \varphi_{ \pm}
$$

with probabilities $|\alpha|^{2}$ or $|\beta|^{2}$, respectively, and we have derived the collapse postulate and Born rule for the spin measurement from the collapse postulate and Born rule for the discretised measurement of position.

In reality, the theoretical description of the experiment will be more complex. For one thing, while we can expect the bulk, call it $\varphi_{++}$, of $\varphi_{+}$to be concentrated on one half of the screen (and similarly the bulk of $\varphi_{-}$), it will have tails spreading out to the 'wrong' half of the screen, say

$$
\varphi_{+}=\varphi_{++}+\varphi_{+-} \quad\left(\text { and } \quad \varphi_{-}=\varphi_{--}+\varphi_{-+}\right) .
$$

Assuming for simplicity

$$
\int\left|\varphi_{+-}\right|^{2}=\int\left|\varphi_{-+}\right|^{2}=: \varepsilon \text { and } \int\left|\varphi_{++}\right|^{2}=\int\left|\varphi_{--}\right|^{2}=1-\varepsilon,
$$

then applying the collapse postulate and Born rule to detecting the electron on the screen yields

$$
\alpha|+\rangle \otimes \varphi_{+ \pm}+\beta|-\rangle \otimes \varphi_{- \pm}
$$

(to be suitably renormalised), with probabilities

$$
|\alpha|^{2}(1-\varepsilon)+|\beta|^{2} \varepsilon \quad \text { or } \quad|\alpha|^{2} \varepsilon+|\beta|^{2}(1-\varepsilon),
$$

respectively.

\footnotetext{
${ }^{1}$ Note this might or might not coincide with the upper or lower half of the screen, as it depends on both the sign of the gradient and the polarity of the magnetic field.
} 
This yields a much more general transformation of the spin state of the electron (which is the degree of freedom we are measuring). Indeed, the spin of the electron is still entangled with its spatial degrees of freedom, and the spin part of the electron is collapsed to an improper mixture: either

$$
|\alpha|^{2}(1-\varepsilon)|+\rangle\left\langle+\left.|+| \beta\right|^{2} \varepsilon \mid-\right\rangle\langle-|
$$

or

$$
|\alpha|^{2} \varepsilon|+\rangle\left\langle+\left.|+| \beta\right|^{2}(1-\varepsilon) \mid-\right\rangle\langle-|
$$

(suitably renormalised).

One realises that even more general transformations on the spin state of the electron can be induced by a detection on the screen, if one considers that the Stern-Gerlach magnetic field itself is not 'ideal' (in order to satisfy the Maxwell equations, it cannot be perfectly homogeneous in the directions perpendicular to that of measurement). Or, indeed, if one considers that one could have chosen, at least in principle, any other unitary coupling between the spin and position degrees of freedom of the electron before proceeding to the detection on the screen.

This illustrates the general phenomenology of measurements in quantum mechanics, as well as their general theoretical description: any physically sensible transformation on the state of the system (a 'completely positive' map) can always be obtained by suitable interaction with some ancilla (whether microscopic or macroscopic), followed by an application of the standard collapse postulate and Born rule on the ancilla. (As in the example, what is tested on the ancilla need not be one-dimensional projections. $)^{2}$

\section{Von Neumann's insolubility argument}

In 1932, von Neumann published his famous discussions of the measurement process, in chapter VI of his Mathematical Foundations of Quantum Mechanics (von Neumann 1932). ${ }^{3}$ The first two sections set up the problem

\footnotetext{
${ }^{2}$ For a comprehensive discussion in the language of POV measures, see e.g. Busch, Grabowsky and Lahti (1995).

${ }^{3}$ Page references are to the 1955 translation (which I have mostly tacitly amended).
} 
and discuss composite systems, then von Neumann opens section 3 with announcing an argument 'to exclude an often proposed possibility for explaining the statistical character of process 1' (p. 437). Von Neumann's 'process 1' (defined on p. 351) is the collapse upon measurement of the quantum state of a system.

The explanation von Neumann is targeting appears to be the following. Assume that the measuring apparatus is initially in some appropriate mixed state $\rho$. For the sake of a vivid example, we could imagine that instead of coupling the spin of the electron to the spatial degrees of freedom of the electron, we couple it directly to a macroscopic 'pointer', and that initially we are not able to prepare a pure wavefunction $\varphi$ for the pointer, but only a proper mixture

$$
\rho=\int_{\Lambda} w_{\lambda} \rho_{\lambda} d \lambda
$$

where each $\rho_{\lambda}$ corresponds itself to a wavefunction $\varphi_{\lambda}$ localised within the spread of the original $\varphi$. For any initial (pure) state $|\psi\rangle\langle\psi|$ of the system, the final state evolving unitarily from the initial $|\psi\rangle\langle\psi| \otimes \rho$ will also be mixed:

$$
U(|\psi\rangle\langle\psi| \otimes \rho) U^{-1}
$$

The question is: could our ignorance of the initial state of the apparatus explain the final mixture of apparently collapsed states?

This idea (before he proceeds to refute it) is formulated by von Neumann as follows (pp. 437-438):

Let $I$ be the observed system, $I I$ the observer. [...] The statistical character comes from the fact that while indeed $I$ was in a homogeneous state ${ }^{4}$ before the measurement, $I I$ instead was a mixture - and the character of mixture of $I I$ has 'infected' $I+I I$ during the course of the interaction, in particular has made a mixture of the projection in $I$. That is: the result of the measurement is

\footnotetext{
${ }^{4}$ The 1955 translation has ' $I$ was in a (unique) state', but the German 'einheitlich' is used by von Neumann explicitly as a synonym for 'rein', i.e. 'pure', in which case it is generally translated as 'homogeneous' (see e.g. the definition of a 'homogeneous or pure' expectation functional on p. 307). Clearly, von Neumann here means a pure state, represented by a projection $|\psi\rangle\langle\psi|$.
} 
indeterminate because the state of the observer before the measurement is not precisely known.

Now, is it 'conceivable that such a mechanism works' (p. 438)? We would need to construct a Hilbert space $\mathcal{H}$ for the 'observer', together with a unitary evolution $U$ for the total system, ${ }^{5}$ an initial state $\rho$ on $\mathcal{H}$, and a finest ignorance-interpretable decomposition

$$
\rho=\int_{\Lambda} w_{\lambda} \rho_{\lambda} d \lambda
$$

of $\rho$, such that for any initial state

$$
|\psi\rangle=\alpha|+\rangle+\beta|-\rangle
$$

of the system, there is a partition $\Lambda=\Lambda_{+} \cup \Lambda_{-}$, which generally depends on $|\psi\rangle$, such that

$$
\int_{\Lambda_{+}} w_{\lambda} d \lambda=|\alpha|^{2}, \quad \int_{\Lambda_{-}} w_{\lambda} d \lambda=|\beta|^{2}
$$

and for all $\lambda \in \Lambda_{ \pm}$

$$
U\left(|\psi\rangle\langle\psi| \otimes \rho_{\lambda}\right) U^{-1}=| \pm\rangle\langle \pm| \otimes \sigma_{ \pm}
$$

where the $\sigma_{ \pm}$represent distinct outcomes of the measurement.

Von Neumann's own argument to the contrary is that, assuming the relevant decomposition of the final state (12) has the form

$$
\sum w_{n}\left|\psi_{n}\right\rangle\left\langle\psi_{n}|\otimes| \varphi_{n}\right\rangle\left\langle\varphi_{n}\right|
$$

(where the $\left|\psi_{n}\right\rangle$ denote the eigenstates of the measured observable, and the $\left|\varphi_{n}\right\rangle$ are some orthonormal set of states in $\left.\mathcal{H}\right),{ }^{6}$ the weights in this decomposition are uniquely determined by the weights in the orthogonal decomposition

\footnotetext{
${ }^{5}$ Since the evolution of the total system is unitary, the total system needs to be closed, and we need to include in the 'observer' anything with which the system will interact directly or indirectly. That is, we need to include any ancillas, pieces of apparatus, and relevant parts of the environment (including human observers if applicable). For simplicity, we shall usually talk of the 'apparatus' or the 'screen', but we shall tacitly include in it everything else of relevance.

${ }^{6}$ Presumably, the microscopic states corresponding to different readings need to be orthogonal, but will not form a basis of the (very high-dimensional) Hilbert space.
} 
of the initial state $\rho$, and are thus independent of the state $|\psi\rangle$ (in our example, this is inconsistent with (15)). This is true of any decomposition of this form, since the weights are unique up to ordering (pp. 438-439). ${ }^{7}$

\section{$3 \quad$ Insolubility from no-signalling}

We shall now show that the impossibility of obtaining (16) in general follows also (and very simply) from the quantum mechanical no-signalling theorem. The no-signalling theorem (Ghirardi, Rimini and Weber 1980) states that performing a measurement on one of a pair of entangled particles (or any other manipulation, in particular unitary interaction with it) does not affect the probability distributions for results of measurements on the other. That is, while conditionalising on the outcomes of a measurement on one particle in general affects the probabilities for the outcomes of measurements on the other, conditionalising on performing the measurement (or any other manipulation) does not. For example, take two electrons in the singlet state. If we measure spin along the same direction on both particles, we have perfect anti-correlations between the outcomes, but averaging over the outcomes on one side yields back the usual 50-50 distribution on the other side. One easily sees that the same is true also for measurements along different spin directions on the two sides, etc.

${ }^{7}$ Note that, arguably, the claim von Neumann is explicitly ruling out has the form

(a) There exist a measurement scheme $(\mathcal{H}, U, \rho)$ and a finest ignorance-interpretable decomposition of $\rho$ that explain the phenomenological collapse for any $|\psi\rangle$.

However, von Neumann's assumption that the ignorance-interpretable decomposition of the final state has the form (17) essentially constrains this decomposition uniquely, so that allowing the decomposition to depend on $|\psi\rangle$ does not make the claim more general. That is, his proof rules out also the a priori weaker

(b) There exists a measurement scheme $(\mathcal{H}, U, \rho)$ such that for any $|\psi\rangle$ there exists a finest ignorance-interpretable decomposition of $\rho$ that explains the phenomenological collapse.

Both Brown (1986) and Bacciagaluppi (2013) argue that, with the exception of Fine (1970), Brown (1986) and Bassi and Ghirardi (2000), the later generalisations of von Neumann's result rule out claims that are essentially equivalent to (b), and thus establish something stronger than insolubility in the original sense. 
The name 'no-signalling' relates to the case in which the two entangled systems are thought of as spatially distant, but the theorem applies generally to any pair of degrees of freedom in an entangled state. To pick our example from section 1, performing a measurement of the position of an electron after it has left the magnetic field of a Stern-Gerlach apparatus (that is, after the dynamical coupling between the spin and the spatial degrees of freedom of the electron has ended) has no net effect on the probability distributions for any spin variables of the electron.

We shall stick to the Stern-Gerlach example, although the proof will be valid for the general case of measurements performed by letting first a microscopic ancilla interact with the system, and then performing some appropriate manipulation of the ancilla. It will be equally valid for the (less realistic) case in which we assume we can couple directly the observable to be measured with some macroscopic 'pointer', but the measurement is performed on one of a pair of entangled systems (e.g. on Alice's side of an EPR pair). Thus, the result will hold for a wide class of measurements, and will provide copious counterexamples to the claim that the statistical spread of results in a measurement can generally be explained by invoking ignorance of the exact initial state of some macroscopic system.

Assume we try and model, using just the unitary Schrödinger evolution, the interaction between the (microscopic) spatial degrees of freedom of the electron and the (macroscopic) screen, in such a way as to be able to derive the phenomenology of the standard collapse upon detection. In the ideal case, as we have seen, after the electron has passed through the Stern-Gerlach magnet, its state is given by (3), i.e.

$$
|\Psi\rangle=\alpha|+\rangle \otimes \varphi_{+}+\beta|-\rangle \otimes \varphi_{-} .
$$

We now apply the idea of (13)-(16) to this case. We assume there is a decomposition of the state $\rho$ of the screen,

$$
\rho=\int_{\Lambda} w_{\lambda} \rho_{\lambda} d \lambda
$$

and a partition of the index set $\Lambda$,

$$
\Lambda=\Lambda_{+} \cup \Lambda_{-}
$$


(the statistics of results will actually not enter our argument, so that the further condition (15) is immaterial for our purposes), such that for all $\lambda \in$ $\Lambda_{ \pm}$

$$
U\left(|\Psi\rangle\langle\Psi| \otimes \rho_{\lambda}\right) U^{-1}=| \pm\rangle\left\langle \pm|\otimes| \varphi_{ \pm}\right\rangle\left\langle\varphi_{ \pm}\right| \otimes \sigma_{ \pm},
$$

the $\sigma_{ \pm}$representing macroscopic spots on the respective halves of the screen. ${ }^{8}$

Now take any run of the measurement that has produced the result ' + '. The component $\rho_{\lambda}$ of $\rho$ that has led to this result is a state of the screen that will produce the result ' + ', if we let the electron impinge on it (through the appropriate unitary interaction). Similarly, there exists another state of a screen that will produce the result '-', if we let the elctron impinge on it via an appropriate unitary interaction. Of course, we cannot prepare these states of the screen in practice (we could prepare them in principle by reversing the time evolution). But we see that there exist states such that, by choosing to let the electron interact with these states by an appropriate unitary interaction, we can produce at will a spot on the upper or lower half of the screen. But now, given (21), we can also guarantee that the spin state of the electron 'collapses' to the corresponding eigenstate. (Or, if passage through the Stern-Gerlach magnet has produced the entangled state (7), we can guarantee that the spin state of the electron evolves to either (9) or (10), suitably renormalised.)

We see that in principle we can influence the statistics of further measurements on the spin degree of freedom of the electron. But this is impossible, because we have interacted unitarily only with the spatial degrees of freedom of the electron (coupling them to this or that state of the screen), and the no-signalling theorem prevents this from having any effect whatsoever on the spin state of the electron.

Thus, the no-signalling theorem rules out the possibility that an imperfectly known initial state of the screen might explain the collapse of the spin

\footnotetext{
${ }^{8}$ More generally, we can assume that the total system evolves to states that are indistinguishable from the states on the right-hand side of (21), even though they might contain some classical or quantum correlations. These states need not be the same on each run of the experiment, as long as they are recognisable as indicating the given result. We can also allow for slight variation (even co-variation) from one run of the experiment to another both in the initial states $|\Psi\rangle$ and in the measurement scheme $(\mathcal{H}, U, \rho)$, as long as for the correct proportion of runs a condition of the form (21) holds. We can even include a further component $\Lambda_{0}$ in (20), giving rise to 'dud runs' of the experiment.
} 
part of the state of the electron in any Stern-Gerlach measurement (whether ideal or not). Similarly, it rules out the possibility that an imperfectly known initial state of Alice's apparatus might explain the collapse of the state of Bob's particle in an EPR experiment. In sum, the collapse of the state of a quantum system cannot in general be explained through ignorance of the exact microstate of the apparatus, just as von Neumann established.

\section{Concluding remarks}

Although I believe the argument as stated is new, there is a family resemblance with a number of other arguments in the literature, in particular the argument showing that deterministic hidden variables theories must violate locality (in the form of parameter independence) in order to recover the quantum mechanical correlations. In the case of an EPR pair in the Bohm theory, one can see this very easily. Indeed, if Alice knew the initial position of her particle, by choosing appropriately either the sign of the gradient or the polarity of the field she could arrange her Stern-Gerlach magnet in such a way as to obtain spin up or spin down at will, and thus force the opposite result on Bob's side (see e.g. the nice description in Albert (1992), chapter 7). More generally, since deterministic hidden variables have to be contextual in general (Kochen and Specker 1967), in the case of perfect correlations the context dependence for one particle translates into nonlocality across the particle pair. Arguments of this kind are known as algebraic proofs of nonlocality (e.g. Stairs 1983, Heywood and Redhead 1983, Bacciagaluppi 1993). Contrapositively, they yield proofs of indeterminism from the assumption of locality (Conway and Kochen 2006). Note that in this paper we have assumed unitary dynamics throughout and no hidden variables, so locality follows from the no-signalling theorem and need not be assumed separately.

Similar arguments hold also for theories with super-quantum correlations as long as they satisfy the no-signalling condition, e.g. in the case of PopescuRorlich boxes, as pointed out by Jeffrey Bub in this same issue (Bub 2012a; see also Bub 2012b).

Finally, I wish to point out the resemblance with Einstein's own version of the EPR argument (the comparison is made in more detail in Bacciagaluppi 
$\left.(2013)^{9}\right)$. As discussed by Fine (1981) and Howard (1985, 1990), Einstein's own version of the argument very simply considered two alternative measurements on sub-system $A$ of an entangled pair, leaving $B$ in a state of one of two different types. However,

[t]he real state of $B$ now cannot depend on what measurement I perform on $A$

(Einstein to Schrödinger, 19 June 1935; all translations from this letter are by E. Crull and myself). ${ }^{10}$ Thus, quantum mechancal states are not the 'real states' of a system.

The 'separation hypothesis' used by Einstein of course is stronger than the assumption of no-signalling, ${ }^{11}$ and we can illustrate why this needs to be the case by reference to the insolubility scenario. Einstein states explicitly that, if the quantum mechanical description is complete,

[t]he statistical character of the measurement outcomes is accounted for exclusively in terms of the measurement apparatus, or the process of measurement.

If this merely meant the initial state of the measurement apparatus, then we would have von Neumann's situation. ${ }^{12}$ As we have seen, Einstein could then help himself to what Alice could do in principle, and only the locality part of the separation principle would be needed for the contradiction. But if the 'measurement process' is meant to be more general, e.g. a genuinely indeterministic collapse process, then Alice cannot control the outcomes even in principle, and the full separation principle is needed (but one also only

\footnotetext{
${ }^{9}$ Please be aware of a confusing amalgam of text and quotation on pp. 95-96 (introduced after the proofs were corrected).

${ }^{10}$ AHQP, mf. 92, section 2-107; this letter has been recently published (in German) in von Meyenn (2011), vol. 2, pp. 537-539.

${ }^{11}$ In Howard's (1985) analysis, it corresponds to the conjunction of 'separability', i.e. the existence of separate states for the two subsystems, and 'locality', i.e. no-signalling, a distinction which Einstein starts making explicitly by 1946 or 1947.

${ }^{12}$ In the classical analogue that precedes the quantum mechanical discussion in the letter, Einstein talks about 'insufficiently known factors foreign to the described system'.
} 
needs to consider what manipulations Alice can perform in practice). In either case, a theory satisfying the suitable locality or separability condition will fail to reproduce the phenomenological collapse of the state of the distant system (and is perhaps also in this sense 'incomplete').

\section{Acknowledgements}

I wish to thank audiences at Aberdeen, Berlin, Cagliari and Oxford, as well as Arthur Fine and Max Schlosshauer, who heard or read and commented on previous versions of this and connected material. I am particularly indebted to Alex Blum, Martin Jähnert and especially Christoph Lehner for discussions of Einstein's argument and of how it might relate (or not) to von Neumann's. These discussions also helped me redirect my use of the no-signalling theorem to the general case of measurements with ancillas, whether or not there is spatial separation. Finally, I wish to thank Elise Crull, my collaborator on the Leverhulme Trust Project Grant 'The Einstein Paradox': The Debate on Nonlocality and Incompleteness in 1935 (project grant nr. F/00 152/AN), during the tenure of which this paper was written.

\section{References}

Albert, D. Z. (1992), Quantum Mechanics and Experience (Cambridge, Mass.: Harvard University Press).

Bacciagaluppi, G. (1993), 'Critique of Putnam's Quantum Logic', International Journal of Theoretical Physics 32, 1835-1846.

Bacciagaluppi, G. (2013), 'Insolubility Theorems and EPR Argument', European Journal of the Philosophy of Science 3, 87-100. Also http://philsci-archive.pitt.edu/9121/ .

Bassi, A., and Ghirardi, G.C. (2000), 'A General Argument against the Universal Validity of the Superposition Principle', Physics Letters A 275, 373-381.

Brown, H. R. (1986), 'The Insolubility Proof of the Quantum Measurement Problem', Foundations of Physics 16, 857-870. 
Bub, J. (2012a), 'Quantum Correlations and the Measurement Problem', http://arxiv.org/abs/1210.6371 .

Bub, J. (2012b), 'Bananaworld: Quantum Mechanics for Primates', http://arxiv.org/abs/1211.3062 .

Busch, P., Grabowski, M., and Lahti, P. (1995), Operational Quantum Physics Lecture Notes in Physics, Vol. m31 (Berlin: Springer; second, corrected printing, 1997).

Conway, J., and Kochen, S. (2006), 'The Free Will Theorem', Foundations of Physics 36, 1441-1473.

Fine, A. (1970), 'Insolubility of the Quantum Measurement Problem', Physical Review D 2, 2783-2787.

Fine, A. (1981), 'Einstein's Critique of Quantum Theory: The Roots and Significance of EPR', in P. Barker and C. G. Shugart (eds.), After Einstein: Proceedings of the Einstein Centennial Celebration at Memphis State University, 14-16 March 1979 (Memphis: Memphis State University Press), pp. 147-158. Reprinted in The Shaky Game: Einstein, Realism and the Quantum Theory (Chicago: University of Chicago Press), pp. 26-39.

Ghirardi, G.C., Rimini, A., and Weber, T. (1980), 'A General Argument against Superluminal Transmission through the Quantum Mechanical Measurement Process', Lettere al Nuovo Cimento 27(10), 293-298.

Heywood, P., and Redhead, M. L. G. (1983), 'Nonlocality and the Kochen-Specker Paradox', Foundations of Physics 13, 481-499.

Howard, D. (1985), 'Einstein on Locality and Separability', Studies in History and Philosophy of Science 16, 171-201.

Howard, D. (1990), "Nicht sein kann was nicht sein darf", or the Prehistory of EPR, 1909-1935: Einstein's Early Worries about the Quantum Mechanics of Composite Systems', in A. I. Miller (ed.), Sixty-two Years of Uncertainty (New York: Plenum Press), pp. 61-111.

Kochen, S., and Specker, E. P. (1967), 'The Problem of Hidden Variables in Quantum Mechanics', Journal of Mathematics and Mechanics 17, 59-88. 
von Meyenn, K. (ed.) (2011), Eine Entdeckung von ganz außerordentlicher Tragweite: Schrödingers Briefwechsel zur Wellenmechanik und zum Katzenparadoxon, 2 vols. (Berlin and Heidelberg: Springer).

von Neumann, J. (1932), Mathematische Grundlagen der Quantenmechanik (Berlin: Springer, 2nd ed. 1996). Transl. by R. T. Beyer as Mathematical Foundations of Quantum Mechanics (Princeton: Princeton University Press, 1955).

Stairs, A. (1983), 'Quantum Logic, Realism, and Value-definiteness', Philosophy of Science 50, 578-602. 